\title{
Competencia comunicativa y norma en los grados de maestro en Educación Primaria
}

\author{
Ana María Aguilar LóPEZ \\ Universidad de Burgos \\ amaguilar@ubu.es
}

Recibido: $14 / 11 / 2012$

Aceptado: 23/01/2013

\section{Resumen}

El presente artículo analiza las asignaturas que presumiblemente contribuyen a desarrollar el nivel C1 de competencia comunicativa en español de quienes cursan los grados de maestro en Educación Primaria. Así, partiendo de las guías docentes de las mismas, se comprueba en qué medida reflejan el concepto de competencia comunicativa propuesto por el MCER y la enseñanza de la norma lingüística.

Palabras clave: Competencia comunicativa, Español, Norma, Grado de maestro, Nivel C1.

\section{Communicative Competence and Linguistic Norm in Primary Education Degrees}

\begin{abstract}
This paper analyzes the subjects/modules which presumably contribute to develop the communicative competence in Level C1 from the students of Primary Education Degree. So, taking as a starting point their course descriptions, we will check to what extend they reflect the communicative competence concept set by the CEFR and the teaching of linguistic norm.
\end{abstract}

Keywords: Communicative Competence, Spanish, Norm, Primary Education Degree, Level C1.

\section{Referencia normalizada}

AGUILAR LÓPEZ, Ana María (2013): "Competencia comunicativa y norma en los grados de maestro en Educación Primaria”. Estudios sobre el Mensaje Periodístico. Vol. 19, Núm. especial marzo, págs.: 25-33. Madrid, Servicio de Publicaciones de la Universidad Complutense.

Sumario: 1. Introducción: Selección, formulación y justificación del tema de investigación. 2. Metodología: Procedimiento de investigación. 3. Desarrollo: análisis de los datos. 4. Conclusiones e implicaciones derivadas. 5. Referencias bibliográficas.

\section{Introducción: Selección, formulación y justificación del tema de investigación} La dicotomía chomskyana de competencia lingüística y actuación ${ }^{1}$ resultó "de escasa aplicación para los intereses de la enseñanza de la lengua" (Pastor, 2006: 184), aunque, justo es reconocerlo, esta propuesta sirvió de revulsivo para otras nuevas.

El concepto de competencia comunicativa, que circulaba ya desde finales de los años 60 (Llobera et alter: 2000: 10), surgió de la transformación del concepto de lengua, que pasó a entenderse prioritariamente como un instrumento de comunicación, y no como un sistema de unidades descontextualizadas, y de la idea de estudiar la lengua desde el uso. Si bien, no fue hasta 1971 cuando Dell Hymes lo abordó explícita-

${ }^{1}$ Entiéndase por competencia lingüística el conocimiento tácito, un tanto inconsciente, pues es en parte innato, que tiene el hablante-oyente ideal sobre la estructura de la lengua y por actuación el uso que dicho hablante-oyente ideal hace de la lengua en situaciones concretas y a través del cual manifiesta su competencia lingüística. 
mente en "On communicative competence" ${ }^{2}$ donde, partiendo de la revisión del concepto de competencia lingüística de Chomsky, proponía el de competencia comunicativa, mucho más amplio. A pesar de todo, estos cuarenta años no han pasado en balde y dicho concepto se ha ido perfilando con las aportaciones surgidas desde diferentes ámbitos (gramática del texto, pragmática, didáctica de la lengua, análisis del discurso...) hasta llegar a la propuesta que el Consejo de Europa (2002) hace en el Marco Común Europeo de Referencia para las Lenguas: aprendizaje, enseñanza y evaluación (MCER). Según este documento integran las competencias comunicativas las lingüísticas, la sociolingüística y las pragmáticas. A su vez, dentro de cada componente es posible catalogar otras subcompetencias, conocimientos, destrezas y habilidades. El concepto de competencia comunicativa que propone el MCER vendría a corresponderse con el esquema del Gráfico 1 .

Gráfico 1. Esquema de las competencias incluidas en la competencia comunicativa según el MCER (2002)

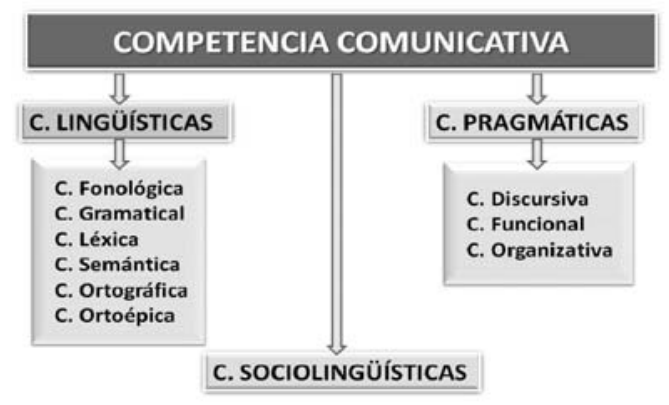

La desmembración del concepto de competencia comunicativa es importante, ya que, como Cenoz (Sánchez y Santos, 2004: 461) señala, "tiene implicaciones pedagógicas a diferentes niveles: a) los objetivos de aprendizaje; b) las estrategias de enseñanza y la autonomía en el aprendizaje y c) la evaluación". Por consiguiente, conocer la taxonomía de los componentes que lo integran nos sirve, entre otras cosas, para asentar unas bases sobre las que se apoye la docencia de la lengua, puesto que, en opinión de Susana Pastor (2006: 183), para desarrollar la competencia comunicativa, debemos acudir a una docencia que incluya todos sus componentes, que atienda a las necesidades comunicativas de los estudiantes y que recurra a interacciones comunicativas realistas y significativas.

Tenemos claro, por tanto, qué contenidos debemos trabajar al enseñar lengua, pero ¿a qué nivel debemos hacerlo en la universidad? Por lo que respecta al alumnado que cursa el grado de maestro en Educación Primaria, según se estipula en la ORDEN $E C I / 3857 / 2007$ (BOE, 312, 2007: 53748), ha de alcanzar, finalizados sus estudios, el nivel $\mathrm{C} 1$ en el dominio de la lengua castellana y, en caso de que exista, en la lengua oficial de la Comunidad Autónoma en la que se cursen. Las alusiones al nivel C1 nos remiten directamente, una vez más, al MCER, pues es este documento el que establece seis niveles posibles de desarrollo de la lengua por parte de los usuarios, a saber: A1 (acceso) y A2 (plataforma), que se corresponden con un usuario que domina la lengua a un nivel básico, B1 (umbral) y B2 (avanzado), que los asocia a un usuario "independiente" en el uso de la lengua objeto, y C1 (dominio operativo eficaz) y C2 (maestría), que son los niveles que cabe esperar en un usuario "competente" de la lengua.

2 En 1971 Dell Hymes presentó esta propuesta a un congreso y en 1972 se publicó como artículo. 
Con esta investigación, pretendemos detectar en qué medida las asignaturas relacionadas con la lengua española de los grados de maestro en Educación Primaria retoman y perpetúan el concepto de competencia comunicativa para desarrollar el nivel $\mathrm{C} 1$ en el dominio de la lengua española.

De este objetivo principal, se deriva otro secundario: comprobar si dichas asignaturas instruyen en el conocimiento de la actual norma culta española. Según aclara César Hernández (web) "las normas lingüísticas se consideran como conjuntos de realizaciones regulares de la lengua [...] sobre todas ellas se superpone una que llega a ser la dominante, porque es la aceptada colectivamente como instrumento comunicativo de prestigio lingüístico o extralingüístico y es la que recibe una valoración positiva de sus hablantes". Esa norma que llega a imponerse, es la lengua culta estándar que, por su prestigio se acepta como modelo por los hablantes, prescinde de vulgarismos, dialectalismos y regionalismos, así como de los rasgos informales y se emplea para transmitir mensajes científicos, técnicos, estéticos o prácticos, pues, no en vano es la lengua propia de la cultura de un pueblo. Para César Hernández (ibíd.) la norma culta del español estándar es la lengua que debe enseñarse y en la que se debe enseñar, siendo obligación del profesorado, independientemente de las asignaturas que imparta, contribuir a que sus alumnos la dominen. Aunque, evidentemente, si no se conoce es imposible enseñarla.

En consecuencia, consideramos primordial que el alumnado de magisterio en general aprenda a expresarse correctamente durante su formación tanto desde un punto de vista normativo, puesto que los maestros son los encargados de enseñar la lengua a los ciudadanos del mañana, como comunicativo, ya que, como mediadores que son entre los contenidos y los niños, deben saber explicar lo que quieren transmitir y hacerlo adecuadamente a la norma y al contexto en el que están inmersos. De ahí que la consecución, por parte de los estudiantes de magisterio, del nivel C1 en lengua española, se correlacione con una formación laboral de mayor calidad.

Por otro lado, son numerosas las voces que desde la etapa educativa anterior a la universitaria (ESO) han alertado sobre las deficiencias expresivas que tiene el alumnado que la cursa y sobre las repercusiones que conlleva, según César Hernández (Ibíd.) "Hay que lograr que todo hispanohablante, al terminar sus estudios obligatorios, sepa expresarse con fluidez, precisión y agilidad en la lengua estándar". Lógico es pensar que, si no todo, parte de ese alumnado con carencias expresivas, accederá a la universidad, responsable entonces de compensar dichas carencias para que no supongan un lastre en el desarrollo de las capacidades de los discentes.

Una vez justificada la selección de nuestro tema de estudio, partimos de la formulación de las siguientes hipótesis que la investigación podrá confirmar o refutar:

Puesto que los grados de maestro en Educación Primaria han de dotar de una formación que garantice el desarrollo del nivel $\mathrm{C} 1$ :

1. La mayoría de las universidades ofertará asignaturas relacionadas con la lengua española.

2. Dichas asignaturas tendrán un peso importante en el plan de estudios.

3. Las asignaturas relacionadas con contenidos de la lengua española: no reflejarán el concepto de competencia comunicativa propuesto por el MCER. 
4. Darán prioridad a los contenidos relacionados con la competencia lingüística.

5. Incluirán alusiones actualizadas a las desviaciones de la norma.

\section{Metodología: Procedimiento de investigación}

Dadas las limitaciones de esta investigación, no se analiza todo el universo objeto de estudio, esto es, todas las universidades españolas, sino una muestra representativa razonablemente ajustada a la totalidad del universo. Así, de las 77 universidades españolas que el Ministerio de Educación, Cultura y Deporte reconoce en su página web, hemos analizado 31 , lo que supone un $40 \%$ del total. El criterio seguido para la selección ha sido incluir entre ellas al menos una de cada comunidad autónoma. En el caso de comunidades autónomas con más de una universidad, la elección ha sido aleatoria.

Así mismo, la técnica empleada en la recogida de datos es la de Análisis de contenido que, en palabras de María José González (1997: 41), es aquélla en la que "Los datos recogidos y analizados proceden de medios de comunicación, periódicos, cartas, etc.", en nuestro caso, los datos se extraen de las guías docentes de las asignaturas ofertadas en los grados de maestro en Educación Primaria que aparecen publicadas en Internet en noviembre de 2012 y que se relacionan directamente con la mejora de la competencia comunicativa del alumnado que las cursa.

Hemos optado por una metodología observacional, pues según Serra et alter, (2000: 77) "los estudios observacionales son imprescindibles para orientar a los investigadores en la profundización de un aspecto de estudio [...] o de aquellos aspectos no explorados todavía". Además, este método implica un mínimo intrusismo por parte del investigador en la situación analizada y facilita un análisis exhaustivo de los datos recopilados, que son, sobre todo, descriptivos.

Al objeto de recoger los datos que nos son necesarios y sistematizar la información relativa a las hipótesis, hemos diseñado dos instrumentos: el primero de ellos, la Tabla 1 , es de carácter general y permite codificar la información relativa a las hipótesis 1 y 2 .

Tabla 1. Instrumento para sistematizar información sobre las hipótesis 1 y 2

\begin{tabular}{|c|c|c|c|c|c|c|c|c|c|}
\hline \multirow{3}{*}{$\mathrm{N}^{\mathrm{o}}$} & \multirow{3}{*}{$\begin{array}{l}\text { Univer. } \\
\text { /Campus }\end{array}$} & \multicolumn{8}{|c|}{ ASIGNATURAS } \\
\hline & & \multirow{2}{*}{$\begin{array}{l}\text { Can- } \\
\text { tidad }\end{array}$} & \multirow{2}{*}{ Nombre } & \multicolumn{2}{|c|}{ Guía } & \multirow{2}{*}{ Tipo } & \multirow{2}{*}{ Créditos } & \multirow{2}{*}{ Curso } & \multirow{2}{*}{ Idioma } \\
\hline & & & & Sí & No & & & & \\
\hline \multicolumn{10}{|l|}{1} \\
\hline & & & & & & & & & \\
\hline 2 & & & & & & & & & \\
\hline
\end{tabular}

Al registrar los datos de universidad y del campus, cubrimos los casos en los que una misma asignatura contenga diferentes guías didácticas según el campus en el que se imparta.

Respecto a las asignaturas tendremos en cuenta aquéllas que cumplan los siguientes requisitos: asignaturas que se impartan en el presente año académico: 2012-2013, que no pertenezcan a una mención específica, se refieran a la lengua española o castellana y que incluyan contenidos específicos relacionados con la mejora de la competencia comunicativa del alumnado que las cursa, lo que supone eliminar aquellas 
asignaturas cuyos contenidos se orienten total o mayoritariamente a la didáctica, al desarrollo del lenguaje por parte de los niños, etc. Ello no obsta para que haya asignaturas cuya denominación aluda a la enseñanza o aprendizaje de la lengua española, pero trabajen, a juzgar por los contenidos de la guía, la competencia comunicativa del alumnado de magisterio que las cursa.

Para obtener datos que nos orienten sobre las hipótesis 3,4 y 5 , se ha diseñado un segundo instrumento que incluye la información de la Tabla 2. Como se aprecia, se han tenido presentes todas las sub-competencias mencionadas por el MCER, si bien, se han emparejado algunas por la proximidad que pueda existir entre los contenidos que se incluyen en ambas.

Respecto a la norma se pretende registrar solamente aquéllas alusiones explícitas a usos incorrectos, es decir, no se tienen en cuenta las alusiones a la norma como concepto lingüístico, como tampoco se considera si dichas alusiones son teóricas exclusivamente o si también se trabajan desde la práctica. Para saber si estas referencias son actuales, se revisa si en la bibliografía de la guía docente se incluyen documentos normativos de la RAE como la Nueva gramática de la lengua española (2009).

Tabla 2. Instrumento para sistematizar la información sobre las hipótesis 3, 4 y 5

\begin{tabular}{|c|c|c|c|c|c|c|c|c|c|}
\hline \multirow{2}{*}{$\begin{array}{c}\text { C.Socio- } \\
\text { lingüística }\end{array}$} & \multicolumn{2}{|c|}{ C. Pragmática } & \multicolumn{5}{c|}{ C. Lingüística } & \multicolumn{2}{c|}{ Norma } \\
\cline { 2 - 8 } & $\begin{array}{c}\text { Discur. } \\
\text { Organiza. }\end{array}$ & $\begin{array}{c}\text { Funcio- } \\
\text { nal }\end{array}$ & $\begin{array}{c}\text { Léxica } \\
\text { Semán. }\end{array}$ & \multicolumn{2}{|c|}{ Gramática } & Ortogra- & $\begin{array}{c}\text { Fonética } \\
\text { Ortoépica }\end{array}$ & $\begin{array}{c}\text { Desvia- } \\
\text { ciones }\end{array}$ & RAE \\
\hline & & & & & & & & & \\
\hline & & & & & & & & & \\
\hline
\end{tabular}

\section{Desarrollo: análisis de los datos}

Como era previsible, durante la recogida de datos hemos encontrado universidades que ofertan una misma asignatura que se imparte en diferentes campus, en algunos casos dicha asignatura se asocia a una sola guía didáctica y en otros a diferentes guías didácticas, de cumplirse esta segunda situación se analizan todas las guías docentes de la misma asignatura.

En los casos en los que se han encontrado asignaturas cuya denominación puede encajar en el perfil de nuestra investigación, pero no se han localizado o no están disponibles las guías docentes de las mismas, dichas asignaturas computan para el análisis de los datos de la Tabla 1, pero no se tienen en cuenta para el análisis de datos de la Tabla 2.

Finalmente el corpus de estudio sobre el que aplicamos el análisis es el que aparece en la Tabla 3. Según se aprecia, un $6,4 \%$ de las universidades analizadas no parecen ofertar el grado de maestro en Educación Primaria, 8 de las 31 universidades del estudio, (el 25,8\%), no ofertan en 2012 asignaturas que se centren en los contenidos de la lengua española o en las actividades de la lengua. En cambio un $67,7 \%$ de las universidades sí ofertan una $(41,9 \%)$ o más de estas asignaturas $(25,8 \%)$. Tan sólo no obtenemos las guías didácticas de 7 asignaturas de 5 universidades, éstas son: Lengua y Literatura (Universidad de Extremadura), Habilidades Lingüísticas y Comunicativas (Universidad de Alcalá), Lengua española y comunicación I y II (Universidad Ne- 
brija), Lengua castellana y Técnicas de la expresión oral y escrita (E.U. de Magisterio Fray Luis de León de la Universidad de Valladolid) y Lengua española y estrategias para la comunicación (Centro adscrito Alberta Giménez de la Universidad de Illes Balears). Si bien, la de Illes Balears y la de Lengua de Valladolid se ofertan en otros campus de los que sí hay guías docentes, por lo que puede que también se apliquen a estos centros aunque no se especifique en las correspondientes páginas web.

Si descontamos aquéllas que no tienen las guías didácticas, tenemos un total de 26 asignaturas, que se corresponden con 29 guías didácticas, pues la asignatura Lengua castellana de la Universidad de Valladolid tiene diferentes guías didácticas en sus campus. De las 26 asignaturas, 14 (un 53,8\%) son de carácter obligatorio, seguidas de las 7 de formación básica $(26,9 \%)$ y de las 4 optativas $(15,3 \%)$. La asignatura Técnicas de expresión oral y escrita de la Universidad de Navarra, queda sin catalogar por no encontrar información referida a esta clasificación. De las 4 asignaturas optativas, tres se refieren a las actividades de la lengua: Técnicas de expresión oral y escrita, Técnicas de comunicación oral y escrita (Universidad de Valladolid) y Lectura y escritura creativa (Universidad Complutense de Madrid). El idioma mayoritario en que se trabajan es el español, aunque la Universidad de A Coruña propone el gallego para impartir la asignatura de lengua española y la del País Vasco ofrece la posibilidad de elegir entre euskera y español para una asignatura destinada a desarrollar la competencia comunicativa en ambas lenguas.

Tabla 3. Elementos de la muestra seleccionada: Corpus de estudio

\begin{tabular}{|c|c|c|c|c|c|c|c|c|c|}
\hline \multirow{3}{*}{$\mathrm{N}^{\circ}$} & \multirow{3}{*}{$\begin{array}{l}\text { Universidad } \\
\text { / Campus }\end{array}$} & \multicolumn{8}{|c|}{ ASIGNATURAS } \\
\hline & & \multirow{2}{*}{ Cant. } & \multirow{2}{*}{ Nombre } & \multicolumn{2}{|c|}{ Guía } & \multirow{2}{*}{ Tipo } & \multirow{2}{*}{ Créd. } & \multirow{2}{*}{ Curso } & \multirow{2}{*}{ Idioma } \\
\hline & & & & Sí & No & & & & \\
\hline \multirow[b]{2}{*}{1} & \multirow[b]{2}{*}{ A Coruña } & \multirow[b]{2}{*}{2} & 1a) Lingua castelán e a súa didáctica & $\mathrm{x}$ & & Obligatoria & 6 & $1^{\circ}$ & gallego \\
\hline & & & 1b) Lingua e liiteratura castelán e a súa didáctica & $\mathrm{x}$ & & Obligatoria & 6 & $2^{\circ}$ & $\begin{array}{l}\text { español/ } \\
\text { gallego }\end{array}$ \\
\hline 2 & Alcalá & 1 & Habilidades lingüísticas y comunicativas & & $\mathrm{x}$ & Optativa & 6 & $\begin{array}{l}\text { No se } \\
\text { dice }\end{array}$ & español \\
\hline 3 & Alicante & 1 & $\begin{array}{l}\text { Lengua y literatura españolas para la enseñanza } \\
\text { primaria }\end{array}$ & $\mathrm{x}$ & & Obligatoria & 6 & $1^{\circ}$ & español \\
\hline \multirow{2}{*}{4} & \multirow{2}{*}{ Burgos } & \multirow{2}{*}{2} & 4a) Lengua española & $\mathrm{x}$ & & Optativa & 5 & $3^{\circ}$ & español \\
\hline & & & 4b) Expresión y comprensión oral y escrita & $\mathrm{x}$ & & Obligatoria & 6 & $1^{\circ}$ & español \\
\hline \multirow{2}{*}{5} & \multirow{2}{*}{ Cantabria } & \multirow{2}{*}{2} & 5a) Lengua española y comunicación I & $\mathrm{x}$ & & Básica & 6 & $1^{\circ}$ & español \\
\hline & & & 5b) Lengua española y comunicación II & $\mathrm{x}$ & & Obligatoria & 6 & $3^{\circ}$ & español \\
\hline \multirow{2}{*}{6} & \multirow{2}{*}{$\begin{array}{l}\text { Complutense de } \\
\text { Madrid }\end{array}$} & \multirow{2}{*}{2} & 6a) Lengua española & $\mathrm{x}$ & & Obligatoria & 6 & $3^{\circ}$ & español \\
\hline & & & 6b) Lectura y escritura creativa & $\mathrm{x}$ & & Optativa & 6 & $2^{\circ}$ & español \\
\hline 7 & Extremadura & 1 & Lengua y literatura & & $\mathrm{x}$ & Obligatoria & 6 & $3^{\circ}$ & español \\
\hline 8 & $\begin{array}{l}\text { Illes Balears } \\
\text { Alberta Giménez } \\
\text { Mallorca }\end{array}$ & 1 & $\begin{array}{l}\text { Lengua española y estrategias para la } \\
\text { comunicación }\end{array}$ & $\mathrm{x}$ & $\mathrm{x}$ & Obligatoria & 6 & $1^{\circ}$ & español \\
\hline 9 & La Laguna & 1 & Lengua española & $\mathrm{x}$ & & Obligatoria & 8 & $2^{\circ}$ & español \\
\hline \multirow{2}{*}{10} & \multirow{2}{*}{ La Rioja } & \multirow{2}{*}{2} & 10a) Lengua castellana para maestros & $\mathrm{x}$ & & Obligatoria & 6 & $1^{\circ}$ & español \\
\hline & & & 10b) Habilidades lingüísticas para la enseñanza & $\mathrm{x}$ & & Obligatorio & 6 & $3^{\circ}$ & español \\
\hline 11 & León & 1 & Enseñanza y aprendizaje de la lengua castellana I & $\mathrm{x}$ & & Obligatoria & 6 & $1^{\circ}$ & español \\
\hline 12 & Murcia & 1 & Comunicación oral y escrita en lengua española & $\mathrm{x}$ & & básica & 6 & $1^{\circ}$ & español \\
\hline \multirow{2}{*}{13} & \multirow{2}{*}{ Navarra } & \multirow{2}{*}{2} & Técnicas de expresión oral y escrita & $\mathrm{x}$ & & No se dice & 6 & $1^{\circ}$ & español \\
\hline & & & Lengua española & $\mathrm{x}$ & & Básica & 6 & $2^{\circ}$ & español \\
\hline 14 & Oviedo & 1 & Lengua y literatura & $\mathrm{x}$ & & Obligatoria & & $1^{\circ}$ & español \\
\hline 15 & País Vasco & 1 & $\begin{array}{l}\text { Desarrollo de la competencia comunicativa- } \\
\text { lengua castellana y lengua vasca- en E.P. I }\end{array}$ & & & Básica & 6 & $1^{\circ}$ & $\begin{array}{c}\text { Español o } \\
\text { euskera }\end{array}$ \\
\hline 16 & $\begin{array}{l}\text { Pontificia de } \\
\text { Comillas }\end{array}$ & 1 & Expresión oral y escrita & $\mathrm{x}$ & & Básica & $4 ' 5$ & $1^{\circ}$ & español \\
\hline 17 & Sevilla & 1 & Lengua española & $\mathrm{x}$ & & Obligatoria & 6 & $3^{\circ}$ & español \\
\hline 18 & Valencia & 1 & Lengua española para maestros & $\mathrm{x}$ & & Básica & 6 & $1^{\circ}$ & español \\
\hline
\end{tabular}




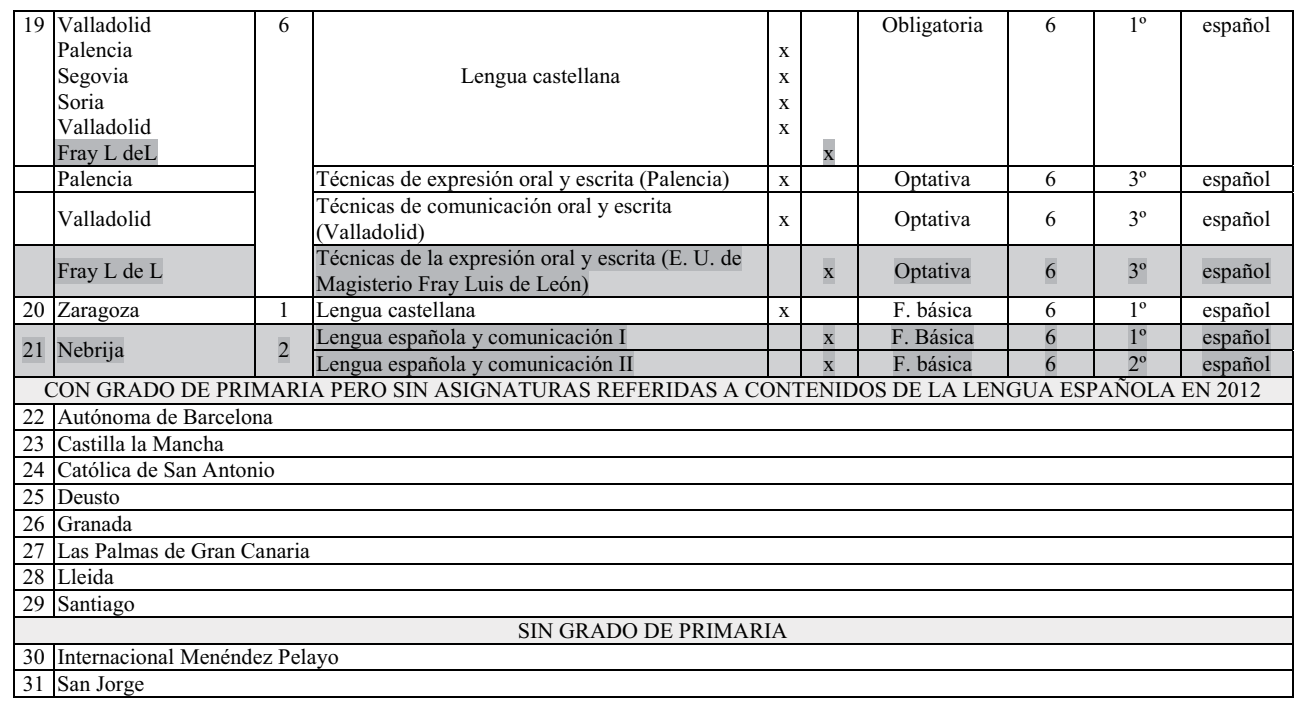

Respecto a los datos registrados a través de la Tabla 2, es preciso aclarar que se han recogido todas las alusiones a estas competencias independientemente de si se refieren a ellas como conceptos o si incluyen contenidos que les son propios. La información con la que hemos completado esta tabla procede sobre todo del apartado de las guías docentes que alude a los contenidos y a la bibliografía, pero no exclusivamente.

Una vez recogidos los datos, comprobamos que las competencias más citadas en las guías son las lingüísticas (se mencionas en 22 de las 29 guías), seguidas de las pragmáticas (20 de las 29) y la sociolingüística (13 de las 29). Dentro de las competencias lingüísticas, la que más referencias tiene es la gramatical, con 20 alusiones en la sintaxis y 19 en la morfología, a éstas le siguen la fonética y ortoépica con 17 alusiones, la léxica y semántica con 16 y la ortográfica con 14. Si nos fijamos en las subcompetencias pragmáticas, la que menos alusiones ha generado es la funcional.

Un 37,9\% de las guías docentes analizadas hacen alusiones explícitas a ninguna, una, dos o tres de las subcompetencias, un $27,5 \%$ menciona cuatro o cinco y otro $34 ' 4 \%$ incluye referencias a seis o siete.

Sobre la norma los datos reflejan que en 13 casos (un 44,8\%) las guías mencionan alusiones a correcciones idiomáticas, de ellos en dos ocasiones no se incluyen referencias bibliográficas, en el resto sí y se incluyen una o varias de las últimas publicaciones de la RAE en este sentido.

\section{Conclusiones e implicaciones derivadas}

Por los datos obtenidos podemos decir que la primera hipótesis relativa a que la mayoría de las universidades oferta asignaturas relacionadas con los contenidos de lengua española, queda confirmada, incluso aunque no tuviéramos en cuenta las asignaturas a cuya guía docente no hemos podido acceder, seguiría confirmándose.

Respecto al peso que dichas asignaturas tienen en el plan de estudios del grado de maestro en Educación Primaria (Hipótesis 2), podemos concluir que muchas de las 
universidades ofertan más de una asignatura que se relaciona con estos contenidos y que dichas asignaturas son en su mayoría de tipo obligatorio o de formación básica, lo que garantiza una formación mínima y común a todos los discentes en este aspecto. Ahora bien, que dicha formación sea suficiente para desarrollar el nivel C1 en lengua española es otro tema, las guías docentes per se, no pueden dar cuenta de esta información, si bien, el MCER (Consejo de Europa, 2002:18) aclara que, pese a que los niveles (A1, A2, B1, B2, C1 y C2) puedan parecer equidistantes, no es más que una mera percepción, pues a medida que se avanza en el domino de una lengua es preciso dedicarle mayor tiempo a cada nivel, porque los niveles superiores implican mayor complejidad, ampliación y perfección de las actividades, destrezas y conocimientos.

Por lo que respecta a la tercera hipótesis: Las asignaturas relacionadas con contenidos de la lengua española no reflejarán el concepto de competencia comunicativa propuesto por el MCER ha quedado refutada.

Es cierto que la competencia que más alusiones explícitas genera es la lingüística, aunque también es la que tradicionalmente se ha incluido en los programas de lengua española e incluye más subcompetencias que el resto de competencias, por lo que, quizá, la cantidad de contenidos que abarque sea mayor. Además, disciplinas como la sociolingüística o la pragmática son mucho más recientes, pero desconocemos si este hecho ha podido influir y en qué medida, en la selección de contenidos que hace el profesorado. Por otro lado, las guías didácticas son un documento general, en ocasiones tanto que no permiten predecir contenidos concretos. En este sentido la alusión a los contenidos más lacónica que hemos encontrado es: "Aspectos descriptivos y normativos de los sistemas y subsistemas de la lengua española y de sus usos orales y escritos", no se menciona explícitamente aquí ninguna de las competencias de la competencia comunicativa, pero suponemos que se trabajan. Es decir, el hecho de que no se mencionen las sub-competencias en la guía didáctica, no es garantía de que no se trabajen, del mismo modo que tampoco lo es su mención, puesto que a veces se pueden abordar como concepto. Es decir, al encontrar contenidos como "La pragmática", no sabemos si se alude a ella como disciplina lingüística o a la inclusión de ciertos contenidos sobre ella. Una vez más, en este sentido las guías docentes tienen limitaciones y, precisamente por su carácter general, es imposible averiguar en cuáles de ellas se aluden a estas disciplinas como conceptos o como contenidos que mejoran la competencia comunicativa, pues, aunque es cierto que en algunos casos se puede entrever en otros la incertidumbre y la ambigüedad están latentes, de forma que no es posible a partir de su estudio sacar conclusiones diáfanas a este respecto.

La hipótesis 5: Las asignaturas relacionadas con contenidos de la lengua española incluirán alusiones actualizadas a las desviaciones de la norma, a tenor de los datos obtenidos, también se cumple.

\section{Referencias bibliográficas}

ALONSO HERNÁNDEZ, César (2001): “¿Qué norma enseñar?”, en Cervantes.es: Congresos Internacionales de la Lengua Española. Congreso Internacional de la lengua española, Valladolid, 2001. 
http://congresosdelalengua.es/valladolid/ponencias/unidad_diversidad_del_espanol/1_la_norma_hispanica/hernandez_c.htm [Última fecha de consulta: $8 \overline{\text { de no- }}$ viembre de 2012]

CONSEJO DE EUROPA (2002): Marco común europeo de referencia para las lenguas: Aprendizaje, Enseñanza, Evaluación. Ministerio de Educación Cultura y Deporte. Instituto Cervantes. Madrid, Anaya.

GONZÁLEZ RÍO, María José (1997): Metodología de la investigación social. Alicante, Aguaclara.

BOE, 312, 29 de diciembre 2007: ORDEN ECI/3857/2007, de 27 de diciembre.

LLOBERA et alter (2000): Competencia comunicativa. Documentos básicos para la enseñanza de lenguas extranjeras. Madrid, Edelsa.

PASTOR CESTEROS, Susana (2004): Aprendizaje de segundas lenguas. Lingüistica aplicada a la enseñanza de idiomas. Alicante, Publicaciones Universidad de Alicante.

SÁNCHEZ LOBATO, Jesús y SANTOS GARGALLO, Isabel (Dir., 2004): Vademécum para la formación de profesores. Enseñar Español como Segunda Lengua (L2)/Lengua Extranjera (LE). Madrid, SGEL.

SERRA, Miquel et alter (2000): La adquisición del lenguaje. Barcelona, Ariel Psicología.

\section{Ana María AGUILAR LÓPEZ}

Universidad de Burgos. Facultad de Humanidades y Educación amaguilar@ubu.es

Profesora del Departamento de Didácticas Específicas, Área de Didáctica de la Lengua y la Literatura. 